\title{
Clustering in a One-dimensional Inelastic Lattice Gas
}

\author{
Srdjan Ostojic, Debabrata Panja and Bernard Nienhuis \\ Institute for Theoretical Physics, Universiteit van Amsterdam, \\ Valckenierstraat 65, 1018 XE Amsterdam, The Netherlands
}

(Dated: November 13, 2018)

\begin{abstract}
We analyze a lattice model closely related to the one-dimensional inelastic gas with periodic boundary condition. The one-dimensional inelastic gas tends to form high density clusters of particles with almost the same velocity, separated by regions of low density; plotted as a function of particle indices, the velocities of the gas particles exhibit sharp gradients, which we call shocks. Shocks and clusters are seen to form in the lattice model too, although no true positions of the particles are taken into account. The locations of the shocks in terms of the particle index show remarkable independence on the coefficient of restitution and the sequence of collisions used to update the system, but they do depend on the initial configuration of the particle velocities. We explain the microscopic origin of the shocks. We show that dynamics of the velocity profile inside a cluster satisfies a simple continuum equation, thereby allowing us to study cluster-cluster interactions at late times.
\end{abstract}

PACS numbers: 47.70.Nd, 45.70.Mg, 05.40.-a, 81.05.Rm

\section{INTRODUCTION}

Dynamics of granular fluids has captured a lot of attention from theoretical physicists for the last few years. In a theorist's model, the constituent particles of a granular fluid, usually considered to be hard spheres of finite radii, irretrievably dissipate kinetic energy via inelastic binary collisions and interparticle frictional forces. As a result, unlike the microscopic models for the classical kinetic theory of gases, a granular fluid that is not driven by external forces "cools freely".

Even in the absence of frictional forces, in a stark contrast to hard sphere fluids with elastic inter-particle binary collisions, such a simplified model of granular fluids exhibits complex behaviour at macroscopic scales. In its simplest form, a freely cooling initially homogeneous and isotropic (both in the particles' position and velocity space) inelastic gas spontaneously forms nontrivial structures in the macroscopic velocity as well as in the macroscopic density field of the gas. A large number of studies, mostly from the point of view of inelastic hydrodynamics, have been carried out to understand the onset for the formation of these structures in two and three dimensions (Refs. [1, 2, 3, 4, 5, 6, 7] to cite a few). The (qualitative and quantitative) picture that has emerged from these studies is that in two and three dimensions, the system of inelastic hard spheres suffers from inherent long wavelength linear instabilities. When the system size allows such long wavelengths to be present, these instabilities start to generate inhomogeneities in the macroscopic velocity and the density field of the gas. In the subsequent evolution, these inhomogeneities interact nonlinearly to give rise to macroscopic structures and the entire system evolves into a collection of densely populated clusters that are separated by regions containing particles at very low density $[1,8]$. At late times, the clusters collide in a very complex manner and merge - a phenomenon that is known as coarsening in the literature [9, 10].
The late time evolution of a freely cooling inelastic gas is thus qualitatively completely different from the linear instability mechanisms at early times. However, although a very large number of studies have been devoted to kinetic theory of freely cooling inelastic gases in two and three dimensions, due to the difficulties associated with the nonlinearities in the behaviour of individual clusters and cluster-cluster collisions, a proper theoretical understanding of the long time dynamics of freely cooling inelastic gases has remained elusive. The existing results have only been numerical [3, 9, 10, 11, 12, 13.

At the other extreme, fully analytical solutions have been found for completely inelastic (or "sticky") granular gas (of point particles) in one dimension [14], and it has been shown that the sticky gas in one dimension is described by the Burgers equation in the inviscid limit 14]. In addition, a recent experiment has also observed clustering in a one-dimensional granular gas $\underline{15}$. Due to dimensional reasons, the dynamics of a granular gas in one dimension is qualitatively different from those in two or three dimensions (e.g., vortices cannot form, a strict ordering of particles from left to right is maintained at all times), but structures are still seen to form in the velocity and as well as in the density field of the gas [16, 17]. Ben-Naim et al [17] have studied the formation of these structures, and have conjectured that at the long times, the behaviour of a one-dimensional inelastic gas should be the same as that of the sticky gas.

Our purpose in this paper is to unravel some of these long-time phenomena at a more microscopic level in a simple one-dimensional lattice model that has been introduced in Ref. 18, 19]. In this model, one considers a system of $N$ particles on the integral lattice positions (denoted by $k$ ) of a ring of size $N$ with initial velocities chosen randomly from a uniform distribution in $[-1,1]$. The ordering of the particles is maintained at all times; however, since the particles do not move in this lattice model, the velocities of the particles are not the time 
derivative of their positions. Instead, $v_{k}$, the velocity of the $k$ th particle, is simply a scalar quantity associated with the $k$ th particle, and $v_{k}$ changes only when the $k$ th particle participates in a collision with one of its neighbours according to the following collision rule. At a collision between $k$ th and $(k+1)$ th particles at any time, the post-collisional velocities $v_{k,(k+1)}^{(+)}$are related to their pre-collisional velocities $v_{k,(k+1)}^{(-)}$by

$$
v_{k,(k+1)}^{(+)}=v_{k,(k+1)}^{(-)} \pm \frac{1+r}{2}\left[v_{(k+1)}^{(-)}-v_{k}^{(-)}\right],
$$

where $0 \leq r \leq 1$ is the coefficient of restitution. Time is measured by the average number of collisions per particle in this lattice model.

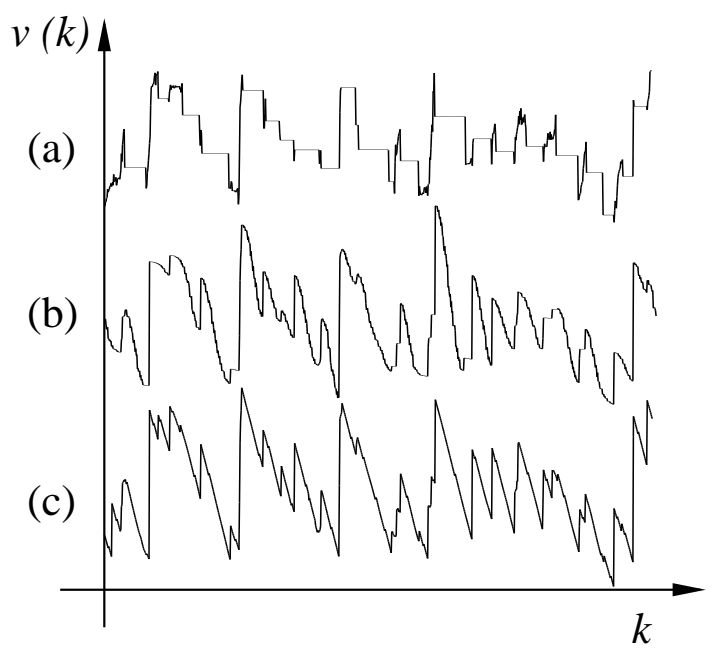

FIG. 1: Comparison of the shock locations on a ring of size $N$ as a function of particle positions $k, k=1,2, \ldots, N$ : (a) a snapshot of one-dimensional inelastic gas 17] for $r=0.3$, (b) a similar snapshot of the random lattice model for $r=$ 0 ,(c)a snapshot of the systematic lattice model. All systems had identical initial positions and velocities of the particles. Visual inspection shows that the locations of the (relative) large shocks are aligned almost perfectly. The scale in the $y$-direction is arbitrary.

In this paper, we will consider two variants of this lattice model, namely the "random lattice model" and the "systematic lattice model". Both obey the collision rule (11), but they differ in the way a colliding pair of particles is chosen to update the system. For the random lattice model, at any update, the colliding pair of particles are chosen randomly from all particles that momentarily satisfy the kinetic constraint $v_{k+1}-v_{k}<0$. On the other hand, for the systematic lattice model, the colliding pair of particles are the ones that has the momentary global minimum value of $v_{k+1}-v_{k}$.

The random lattice model without kinetic constraint, has already been studied as part of a larger class of models, the inelastic Maxwell models [20], in which the col- lision frequency is chosen to be independent of the incoming velocities of colliding particles. For that model, it has been analytically shown that correlations develop with a diffusively growing correlation length, which consequently affects the temperature decay rate. In addition, the inelastic lattice model in one-dimension with the kinetic constraint, which we study here, was also analyzed in Refs. 18, 19] in terms of velocity distribution and structure factors 21]. It is however important to realize the difference between these existing results and the ones reported in this paper: the existing results mainly concern global quantities, while in this paper, our main thrust is to study the behaviour of the microscopic inhomogeneities arising from the kinetic constraint.

The key feature of both variants of the lattice model with kinetic constraint studied here is that an initial configuration of random velocities of the particles soon develops distinct spatial structures, eventually leading to large positive sharp jumps in the particle velocities intersparsed with relatively smooth variations. We refer to the large positive sharp jumps in the velocity field as shocks and the region between two consecutive shocks, where the velocity variations are smooth, as clusters. These structures have already been observed in [18], but have not been fully analyzed. Formation of shocks and the subsequent dynamics of the clusters make the lattice model interesting on its own, but its relevance is realized only when the locations of its shocks in $v(k)$-profile are compared to those of the one-dimensional inelastic gas [14, 17] (see Fig. 11). By contrast, there are no shocks in the one dimensional random lattice model without kinetic constraints [20].

This paper is organized in the following manner: in Sec. III we discuss the generic features of the lattice model and explore its connections with the onedimensional inelastic gas [14, 17]. In Sec. [II] we analyze the formation of shocks at early times and cluster dynamics at late times. We finally end the paper with a short discussion in Sec. IV]

\section{GENERIC FEATURES OF THE LATTICE MODEL}

A. Phenomenology of shock development and the subsequent dynamics for the random lattice model

To start with, in Fig. 2] we show a time sequence of the velocity profile $v(k)$ for the random lattice model of 10000 particles with $r=0.7$. The initial configuration [Fig. 2(a)] is created by choosing $v_{k}$ randomly from a uniform distribution in $[-1,1]$. As can be seen in Fig. 2(b), velocity correlations set in very rapidly (within 10 collisions per particle). After $10^{4}$ collisions per particle, shocks and clusters can be clearly identified [Fig. 2[(c)]. According to the kinetic constraint, neighbouring particles cannot collide across a shock, and as a result, each cluster evolves independently of the others, until 
two neighbouring clusters collide and coalesce to form a new bigger cluster [this mechanism is illustrated by the evolution of velocity profile of Fig. 2(c) to that of 2(d); Fig. 2(d) corresponds to a state after $10^{6}$ collisions per particle].

From the above phenomenological description, one can identify two separate regimes: the initial homogeneous regime, and the subsequent clustered regime at late times. To make the evolution of the system from one regime towards the other quantitatively more precise, we notice that it corresponds to a symmetry breaking of the probability distribution $P\left(\Delta v_{k}, t\right)$ of relative velocities $\Delta v_{k}=v_{k+1}-v_{k}$. Initially, for each $k$, the distribution of $v_{k}$ is uniform in $[-1,1]$ (i.e., a "box" distribution). Naturally, $P\left(\Delta v_{k}, t=0\right)$ is a piecewise linear distribution on $[-2,2]$ (a "triangular" distribution), symmetric around $\Delta v_{k}=0$. Under the effect of the dynamics, this symmetry is not preserved. This is easily understood from the fact that only the neighbouring particle pairs with $\Delta v_{k}<0$ can collide, and after a collision between the $k$ th and $(k+1)$ th particle, $\Delta v_{k-1}, \Delta v_{k}$ and $\Delta v_{k+1}$ change in a way that, in general, does not preserve the symmetry of $P\left(\Delta v_{k}, t\right)$. As this process continues, from phenomenological considerations, we know that a few shocks remain at late times. Now, since shocks correspond to large values of $\Delta v_{k}$, this means that in the clustered regime, the probability to have a large positive value of $\Delta v_{k}$ is expected to be greater than that of a correspondingly large negative value.

To detect the locations of the shocks by following the evolution of $P\left(\Delta v_{k}, t\right)$ as a function of time, one can identify the location $k$ of a shock by requiring $\Delta v_{k}>$ $C\left|\min _{j} \Delta v_{j}\right|$, where $C>0$ is a constant. The choice of the numerical value of $C$ is arbitrary, and this arbitrariness can be used to tune the minimum value that $\Delta v_{k}$ must have in order to qualify for a shock. Furthermore, if one defines the instant $t_{p}$ when the system passes from one regime to the other by the minimal time where at least one shock becomes visible, then the above requirement can also be used to characterize $t_{p}$. Of course, the precise value of $t_{p}$ depends on the chosen value of $C$.

In the clustered regime, due to inelastic collisions, the amplitude of the velocity profile within each cluster decreases, and the velocities of the particles approach that of the center of mass of the cluster itself. This process continues for a while until two neighbouring clusters, with center of mass velocities $V_{j}$ and $V_{j+1}$ collide and coalesce and the shock separating them disappears [at time $t$, if we number the clusters $j=1,2, \ldots, j_{1}(t)$ from the left to the right, then such a cluster-cluster collision takes place only if $\left.V_{j}>V_{j+1}\right]$. Precisely this mechanism is responsible for making the velocity profile of Fig. 2(c) evolve to that of Fig. 2/d).

At very late times, due to periodic boundary condition, only a single cluster survives. Its amplitude also decreases in time, and the velocities of all particles asymptotically converge to the center of mass velocity of the whole system.
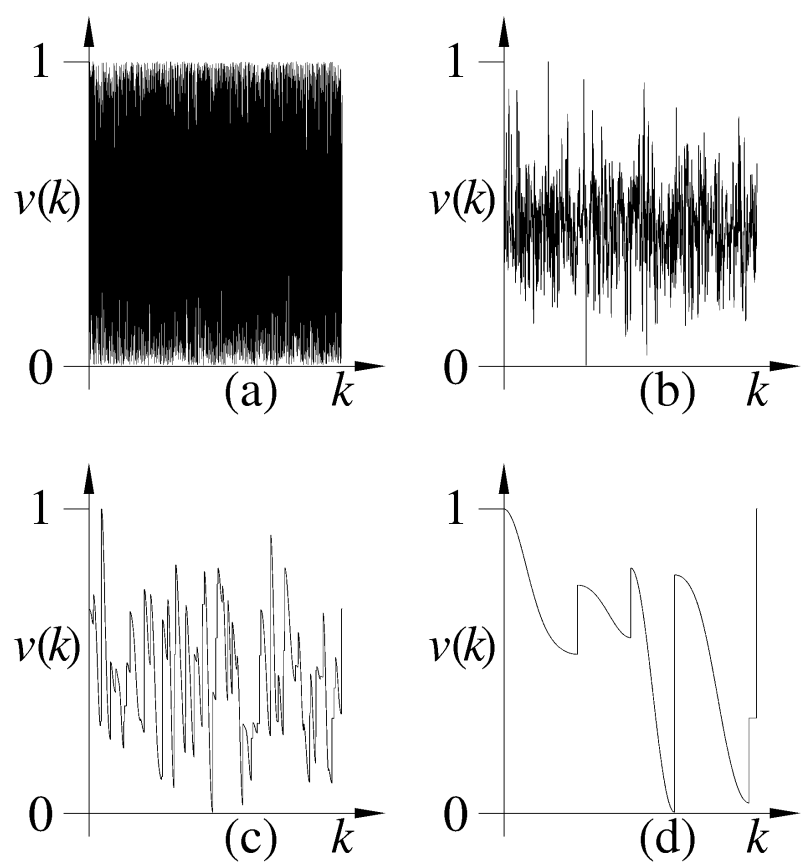

FIG. 2: An example time sequence of shock developments for a system of 10000 particles in the random lattice model: (a) Initial profile (randomly chosen $v_{k}$ from a uniform distribution in $[-1,1])$, (b) after 10 collisions per particle, (c) after $10^{4}$ collisions per particle, (d) after $10^{6}$ collisions per particle. The largest $\left|v_{k_{1}}-v_{k_{2}}\right| \forall k_{1}, k_{2}$ have been scaled to unity in each graph.

\section{B. Dependence on the coefficient of restitution for the random lattice model}

One of the first questions that one can ask for the lattice model is what is the effect of inelasticity on the clustering properties of the system. Clearly, for $r=1$, the collisions are elastic, and upon collision, the velocities of two neighbouring particles are simply interchanged. Such a dynamics cannot lead to clustering or any structure formation.

On the other hand, our simulations show that the lattice model exhibits clustering for any value of $r$ smaller then 1 , and it is only $t_{p}$ defined in Sec. IIA that becomes an increasing function of $r$. The most remarkable observation, however, is that for a given initial velocity configuration of the particles, not only the locations of the shocks persisting at late times are the same for all $r$, but also the macroscopic velocity profiles are nearly independent of $r$. If we consider the macroscopic velocity profile of the lattice model with a coefficient of restitution $r_{1}$ at a time $t_{1}$ well into the clustered regime, then for any $r_{2}>r_{1}$, (originating from the same initial velocity configuration of the particles) there exists a time $t_{2}<t_{1}$, for which the two macroscopic velocity profiles are almost the same (see Fig. 3). Effectively, this implies that for all $r$, the dynamical behaviour of the system is identical 
to that for $r=0$ at late times. We also observe that at intermediate times, the maximum number of shocks (and correspondingly, clusters) in the system are observed for $r=0$ (see Sec. IIA in this regard).
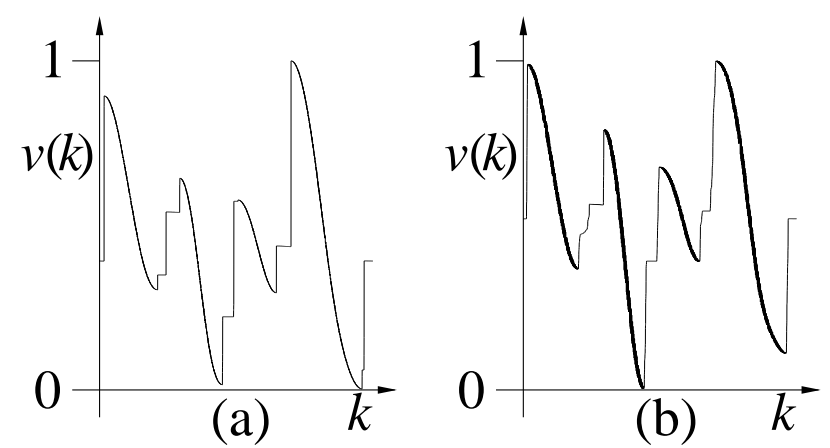

FIG. 3: Velocity profiles for the random lattice model of 5000 particles: (a) for $r=0.7$ after $10^{4}$ collisions per particle (b) for $r=0.95$ and $10^{3}$ collisions per particle. The initial velocity configurations of the particles were the same for both (a) and (b). Once again, the largest $\left|v_{k_{1}}-v_{k_{2}}\right| \forall k_{1}, k_{2}$ has been scaled to unity for both profiles.

\section{Dependence on the order of collisions and on the initial velocity configuration of the particles}

The next important question one can ask is if not $r$, exactly what influences the positions of the shocks? A priori, one expects that the locations of the shocks and the long time dynamics of the system depend on the initial velocity configuration of the particles, as well as on the sequence in which the collisions are performed.

Surprisingly however, the locations of the shocks depend only on the initial configuration of the particles' velocities, e.g, two different random collision sequences result in the same locations of the shocks. On the other hand, the variant of the lattice model (random or systematic) determines the macroscopic shape of the velocity profile inside the clusters. To demonstrate this phenomenon, starting from the same initial velocities of the particles, we simulated both the random and the systematic lattice models and compared the locations of the shocks (see Fig. 11).

\section{Relation between the lattice model and one-dimensional inelastic gas}

The empirical observations in the preceding sections suggest that so long as one is only interested in the velocity profile $v(k)$, a close relation exists between the one-dimensional inelastic gas and the lattice model from the following consideration: the dynamics of a onedimensional inelastic gas for a given initial configuration is completely deterministic — after any collision, the next colliding pair is automatically determined by the instantaneous minimal value of $\left(x_{k+1}-x_{k}\right) /\left(v_{k}-v_{k+1}\right)$, for $v_{k}-v_{k+1}>0$. In this sense, one can view the onedimensional inelastic gas as a variant of the lattice model too, where the colliding particles are chosen in a very complicated manner. Section $\amalg \mathrm{C}$ then indicates that for a given initial configuration of the particles' velocities as a function of the particle index, the locations of the shocks in the one-dimensional inelastic gas and those in the lattice model are the same. That this is indeed the case is numerically verified in Fig. 1]

In our simulations of the one-dimensional inelastic gas, we followed the procedure outlined in Ref. [17], namely that to avoid the inelastic collapse, the collisions were taken to be elastic when the relative velocities between the neighbouring particles are smaller than a given cutoff. Our results confirm the existence of "Burgers" shocks in the $v(x)$ profile [17. More importantly, we note that for the one-dimensional inelastic gas, there is an inverse correspondence of shocks and clusters between this $v(x)$ and $v(k)$ profile - each shock in the $v(x)$ profile [i.e., a large particle density $n(x)]$ corresponds to a cluster in the $v(k)$ profile and the shocks in the $v(k)$ profile correspond to regions with finite gradients in $v(x)$.

\section{ANALYSIS}

\section{A. Development of shocks}

The lesson that we learnt from Sec. III is that the locations of the shocks in this lattice model are essentially determined from the initial configuration of the particles' velocities. This immediately gives rise to the following question: how can one predict the locations of the shocks seen at early times from the initial velocity configuration of the particles?

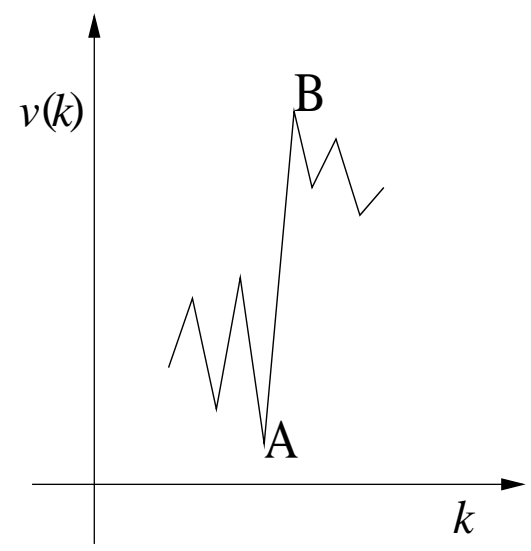

FIG. 4: A schematic diagram of the initial velocity profile for a few particles to explain the development of shocks.

As it turns out, most of the shocks observed in the clustered regime already exist at $t=0$. These are the 
locations marked by large positive jumps in the $v(k)$ profile between two neighbouring lattice sites at $t=0$, surrounded by relatively small velocity variations. To illustrate this point, we present a schematic diagram for such a velocity profile for a few particle in Fig. (4) In the subsequent dynamics at short times, the particles on the right of $\mathrm{B}$ and on the left of A collide with each other and "thermalize" (i.e., the velocity variations reduce due to inelasticity), but this thermalization may not necessarily convert a relatively large $v_{B}-v_{A}>0$ to $v_{B}-v_{A}<0$ quickly enough to make the $A$ and $B$ collide. In other words, such a large positive jump remains preserved and may eventually give rise to a shock.

To check if such a scenario is correct or not, one needs to identify the locations of such large positive jumps in the initial $v(k)$ profile, and contrast them with the observed locations of the shocks at early times. A convenient way to single out such jumps [say, in Fig. 2(a)] is to define a $(2 n+1)$-coarse grained velocity profile $\bar{v}(k)=\sum_{i=k-n}^{k+n} v_{i} /(2 n+1)$ of the particles, which, roughly speaking, can single out one such large jump over the surrounding small velocity variations in a window of $(2 n+1)$ lattice sites in the $\bar{v}(k)$ profile. Having fine-tuned $n$, a surprisingly close match between the observed shock locations at early times and the initial configuration of the particles' velocities can be found (see Fig. 5).

Notice, however, in Fig. 5 that not all the large jumps in the initial configuration of the particles' velocities have turned out to be shocks at a later time. Indeed, in general, whether such an initial large positive jump at a particular location develops into a shock or not really depends on the magnitude of the jump itself in relation to particle velocities in its immediate vicinity, and as well as on the coefficient of restitution $r$. In this regard, the dependence on the coefficient of restitution is not difficult to understand, as $r$ quantifies the "transport of velocity" between two colliding particles. Intuitively speaking, an inelastic collision between two neighbouring particles can be viewed as a combination of dissipation (thermalization) and transport of velocities. For $r=1$ only transport of velocities can take place. At the other limit, for $r=0$, there is no transport of velocities but only dissipation. As a result, for $r=0$, small velocity variations around any large jump thermalize immediately, leading to an early appearance of the clustered regime, and a large number of initial jumps end up becoming shocks. With increasing $r$, some of the initial large positive jumps are eliminated by transport of velocities, and clustering regime, with a smaller number of selected shocks (and clusters), appears later.

The appearance of the shocks at the locations where relatively large positive jumps exist is therefore caused by the thermalization of the smaller velocity variations around these jumps - due to thermalization, the smaller velocity variations become even smaller, but the large positive jumps remain preserved under the dynamics. In

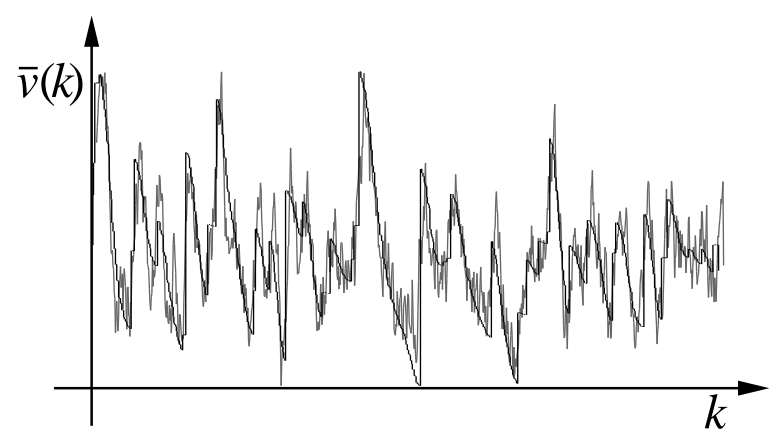

FIG. 5: Correspondence between the coarse grained velocity profiles $\bar{v}(k)(n=7)$ for $r=0$ for the random lattice model of 1000 particles. Gray curve corresponds to $t=0$ and the black curve represents early shocks. The scale in the $y$-direction is arbitrary.

relation to their surroundings, the magnitude of these large jumps thus starts to grow in time. Such a scenario applies independently of the details of the model, and gives a qualitative explanation for the observation that starting from a given initial configuration, shocks appear at the same locations in the $v(k)$ profile for both variants of the lattice model and as well as for the one-dimensional inelastic gas [14, 17]. Moreover, it also clearly demonstrates that the shock formation "instability" in the $v(k)$ profile in all these models is a "relative instability" and not an "absolute" one. Such behaviour has also been found in the (long-wavelength) linear instability of the macroscopic flow field for two- and three-dimensional inelastic gases (see Refs. 1, 4, 7] for example).

\section{B. Cluster dynamics}

\section{Dynamics within a single cluster}

Once the shocks develop, our simulations show that for a given variant of the lattice model, the velocity profile within each cluster has the same characteristic macroscopic shape. For the deterministic lattice model, this shape is simply linear with a negative slope. Although we cannot provide an analytical derivation of this shape, if we interpret the dynamics along the lines that at each updating step of a cluster, two neighbouring particles collide at the location where the $v_{k+1}-v_{k}$ is minimum (which is a mechanism that tries to align all $v_{k+1}-v_{k}$ values to its maximum value within each cluster), then the linear profile appears to be intuitively reasonable.

For the random lattice model, however, the slope of the macroscopic velocity profile is smoothly varying, as can be seen in, e.g., Fig. 2(d) [occasionally, flat profiles can be observed too, but for the time being, we leave them for Sec. IIIB 2. It turns out that the functional form of the smooth macroscopic velocity profile can be obtained 
through a mean-field approach by averaging over an ensemble of random collision sequence realizations within a cluster, as we describe below.

The idea behind this mean-field approach is the following: we consider a cluster of $M$ particles, and denote the velocity of the $k$ th particle at time $t$, averaged over random collision sequences, by $\left\langle v_{k}(t)\right\rangle$. From the very definition of a cluster in the introduction, we assume that $\left\langle v_{k+1}(t)\right\rangle-\left\langle v_{k}(t)\right\rangle<0$ at all times. Thereafter, as we observe from direct simulation measurements that the probability of collisions between any two neighbouring particle pairs are equally likely, in this mean field approach, $\left\langle v_{k}(t)\right\rangle$ is easily seen to satisfy the equation

$$
\begin{aligned}
\left\langle v_{k}(t+1)\right\rangle= & (1-2 p)\left\langle v_{k}(t)\right\rangle \\
& +p\left[\left\langle v_{k}(t)\right\rangle-\varepsilon\left\{\left\langle v_{k}(t)\right\rangle-\left\langle v_{k-1}(t)\right\rangle\right\}\right] \\
& +p\left[\left\langle v_{k}(t)\right\rangle+\varepsilon\left\{\left\langle v_{k+1}(t)\right\rangle-\left\langle v_{k}(t)\right\rangle\right\}\right] .
\end{aligned}
$$

Here, $p$ is an effective probability of a collision between any two neighbouring particles in this mean-field theory and $\varepsilon=(1+r) / 2$. The three terms on the r.h.s. of Eq. (5) respectively originate from the events when the $k$ th particle is not involved in a collision, when there is a collision between the $k$ th and the $(k-1)$ th particle and when there is a collision between the $k$ th and the $(k+1)$ th particle. As Eq. (2) very simply reduces to

$$
\begin{array}{r}
\left\langle v_{k}(t+1)\right\rangle=(1-2 p \varepsilon)\left\langle v_{k}(t)\right\rangle+p \varepsilon\left\langle v_{k-1}(t)\right\rangle \\
+p \varepsilon\left\langle v_{k+1}(t)\right\rangle,
\end{array}
$$

the interesting point to note is that Eq. (3) is the discrete (both in space and time) form of the diffusion equation

$$
\frac{\partial\langle v(k, t)\rangle}{\partial t}=D(r) \frac{\partial^{2}\langle v(k, t)\rangle}{\partial k^{2}},
$$

where mean-field theory predicts $D(r)=p \varepsilon$. One has to keep in mind however that Eq. (4) holds only for monotonically decreasing $\langle v(k, t)\rangle$ as a function of $k$. A similar equation has also been found in the one-dimensional lattice model without the kinetic constraint [20].

To solve Eq. (4), the macroscopic velocity profile of a cluster at time $t_{0}$ can be generally expanded in a Fourier series. Since particle velocities are not transported across the boundaries of a cluster, $\frac{\partial\left\langle v_{k}(t)\right\rangle}{\partial k}$ must vanish at the boundaries of a cluster, the Fourier series contains only terms $\propto \cos [\pi j k]$. The amplitude of the $j$ th such term decreases as $e^{-\pi^{2} j^{2} D(r)\left(t-t_{0}\right)}$, so that for large values of $\left(t-t_{0}\right)$, only the slowest decay mode $j=1$ survives. Hence at long times we expect $\langle v(k, t)\rangle$ to be given by

$$
\begin{aligned}
\langle v(k, t)\rangle & =A(t) \cos \left[\frac{\pi k}{M}\right] \\
& =A\left(t_{0}\right) e^{-\pi^{2} D(r)\left(t-t_{0}\right) / M^{2}} \cos \left[\frac{\pi k}{M}\right] .
\end{aligned}
$$

The mean-field result (5) allows us to compare the halfcosine form of $\langle v(k, t)\rangle$ with the macroscopic velocity profile of the particles within a cluster, as observed in the
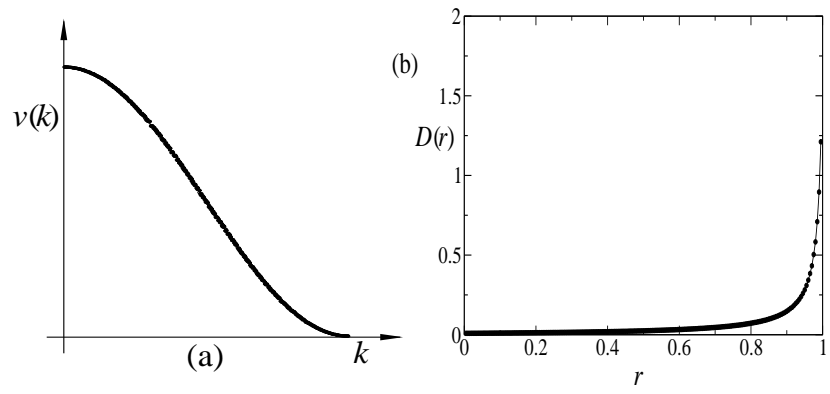

FIG. 6: (a) Comparison of the half-cosine solution (3) [solid line] and simulation data[open circles], the two are completely indistinguishable from each other, (b) Numerically obtained dependence of $D(r)$ on $r$ for the random lattice model. The simulations were carried out for $M=1000$. The scale along the $y$ axis for Fig. (a) is arbitrary. $D(r)$ exhibits a singularity at $r=1$, which is in clear disagreement with the mean-field theory prediction.

simulation for the random lattice model. This comparison is shown in Fig. 6(a) - the fact that we cannot distinguish the simulation data from the half-cosine shape of $\langle v(k, t)\rangle$ as predicted by Eq. (5) is an indication of how well the mean-field approach works to describe the average shape of an isolated cluster. In addition, by following the dynamics from the simulation, we can also obtain an empirical functional form of $D(r)$ [shown in Fig. 6(b)].

There are two features of the $D(r)$ vs. $r$ curve that require further elaboration. The first one of them is that $D(r)$ is an increasing function of $r$, indicating that the amplitude of the cluster $A(t)$ decreases faster as $r$ increases. This observation seems to contradict the fact that the dissipation decreases with increasing $r$. The point to notice however is that the mean-field approach really describes the time evolution within a cluster in which $\frac{\partial\left\langle v_{k}(t)\right\rangle}{\partial k}<0$. From that point of view, the amplitude of the cluster decreases not only from the dissipation, but also from transport of velocities. This is easily seen from the fact that after a collision, the ordering of the magnitudes of the velocities of the colliding particles are simply exchanged, i.e., the particle on the right has a higher velocity, which results in a local positive slope in the $v(k)$ profile. For increasing $r$, transport of velocities become more effective, and thus the negative slope of $\frac{\partial\left\langle v_{k}(t)\right\rangle}{\partial k}$ within a cluster decays to zero faster. Only if $r=1$, the velocities of the particles within an isolated cluster order themselves in an increasing order of magnitude. However, $D(r)$ has a singularity at $r=1$ and the $r=1$ case cannot be treated within the scope of our mean-field theory. Secondly, from the way we presented our mean-field approach, it may seem that the $r$-dependence of $D(r)$ can be obtained through the dependence of $p$ and $\varepsilon$ on $r$. This is actually not true. The mean-field approach (2) neglects fluctuations in the 
particles' velocities around $\langle v(k, t)\rangle$, but in an actual simulation, these fluctuations are very important as they decide the sign of $\Delta v_{k}(t)=v_{k+1}(t)-v_{k}(t)$ for the particles within the cluster and thereby control which collisions are possible and which are not.

\section{Interacting clusters for the random lattice model}

With the background of Sec. IIIB 1 it is now clear that as time progresses, the amplitude of each cluster present in the random lattice model at late times effectively decays exponentially as $\sim \exp \left[-\pi^{2} D(r)\left(t-t_{0}\right) / M^{2}\right]$. Such a decay brings the velocities of the particles within a cluster closer and closer to the centre-of-mass velocity of the cluster itself. Let us assume that at time $t_{0} \gg 1$, there are $j_{1}$ number of clusters present in the whole system of the random lattice model. If we number these clusters by $j$ such that $j=1,2, \ldots, j_{1}$ and denote the number of particles within the clusters, the amplitudes of the clusters and their velocities of their centre of masses respectively by $M_{j}, A_{j}$ and $V_{j}$, then with increasing time, we observe the following dynamics: (i) when $V_{j-1}<V_{j}<V_{j+1}$, the shocks on the left and on the right of the $j$ th cluster cannot respectively be smaller than $V_{j}-V_{j-1}>0$ and $V_{j+1}-V_{j}>0$. In that case, the amplitude $A_{j}$ simply decreases to 0 , forming a "flat" velocity profile (see e.g., Fig. [3). (ii) On the other hand, when $V_{j}>V_{j+1}$, the magnitude of the shock between the $j$ th and the $(j+1)$ th clusters decreases to zero, and the two clusters coalesce together to form a new bigger cluster in a finite time. For a given configuration of clusters at time $t$, the mean-field theory of Sec. IIIB 1 provides us with a way to measure the time $t^{\prime}=t+\Delta t_{\text {min }}$ of the first coalescence of two clusters in the system. In fact, $\Delta t_{\min }=\min _{j} \Delta t_{j}$, where for each pair of neighbouring clusters $(j, j+1)$ with $V_{j}-V_{j+1}>0, \Delta t_{j}$ is obtained by solving the equation

$$
\begin{aligned}
& A_{j+1}\left(t_{0}\right) \exp \left[-\pi^{2} D(r) \Delta t_{j} / M_{j+1}^{2}\right] \\
& \quad+A_{j}\left(t_{0}\right) \exp \left[-\pi^{2} D(r) \Delta t_{j} / M_{j}^{2}\right]=V_{j}-V_{j+1}
\end{aligned}
$$

Due to the fact that Eq. (6) is transcendental in nature, a closed form analytical solution for $\Delta t_{j}$ is impossible to obtain, let alone the value of $\Delta t_{\min }$. Nevertheless, Eq. (6) provides us with a glimpse of how complicated it is to theoretically study the cluster-cluster collisions and coalescence in the random lattice model, and in general, in inelastic gases. If two clusters $l$ and $l+1$ are the first ones to coalesce (at time $t_{0}+\Delta t_{1}$ ) in the random lattice model, then a new cluster with $M_{l}+M_{l+1}$ particles and center of mass velocity $\left(M_{l} V_{l}+M_{l+1} V_{l+1}\right) /\left(M_{l}+M_{l+1}\right)$ is formed. At time $t_{0}+\Delta t_{1}$, the shape of the new cluster is different from a half-cosine, but as the mean-field theory of Sec. IIIB 1 suggests, very soon the shape of the new cluster converges to a half-cosine, unless the newly formed cluster collides with another one in the meantime.

\section{A "hydrodynamic" description of the random lattice model}

So far, we have analyzed the system-wide properties of shocks and clusters. We have also seen that there exists an effective dynamics in terms of diffusion equations (4) and (5) within a single cluster. Based on this collected wisdom on the random lattice model so far, one can naturally ask if it is possible to express the systemwide properties of shocks and clusters in terms of an effective ("hydrodynamic") equation.

It turns out that indeed such an equation can be constructed for the random lattice model:

$$
\frac{\partial v}{\partial t}=D(r)\left[\Theta\left(-\nabla^{+} v\right) \nabla^{+} v-\Theta\left(-\nabla^{-} v\right) \nabla^{-} v\right]
$$

where $\Theta$ denotes a unit step function, $\left(\nabla^{+} v\right)_{k}=v_{k+1}-v_{k}$ and $\left(\nabla^{-} v\right)_{k}=v_{k}-v_{k-1}$ are the discrete gradients operating to the right and left respectively and $\frac{\partial}{\partial t}$ the discrete time gradient. It is important to stress that in a numerical implementation of Eq. (7), all pairs of particles with negative relative velocities collide simultaneously in a unit timestep. The main difference between the numerical implementation of Eq. (7) and the random lattice model lies in the order of the collisions, but in the light of the previous results, we expect to find the shocks at the same locations. Moreover, inside a given cluster, (7) reduces to (4), so that the cluster velocity profiles should be the same as in the random lattice model.

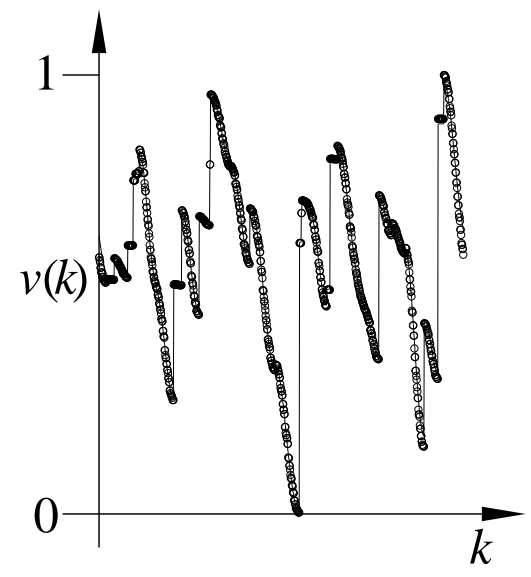

FIG. 7: Comparison of the macroscopic velocity profile (solid curve) predicted by Eq. (7) with the corresponding actual simulation data (open circles) of the random lattice model for $r=0$. The simulations were carried out for 1000 particles and the largest $\left|v_{k_{1}}-v_{k_{2}}\right| \forall k_{1}, k_{2}$ have been scaled to unity both for the actual simulation data and the solid curve.

Indeed, we find that the macroscopic velocity profile obtained numerically from Eq. (7) compares very well with the actual computer simulation results of the random lattice model (see Fig. 7), as expected. 


\section{DISCUSSION}

In this paper, we have extensively studied structure formation and their dynamics in a one-dimensional inelastic lattice model for granular gases. These structures appear in the form of clusters separated by shocks. The locations of the shocks in this lattice model at early times are decided from the initial configurations of the particles' velocities in a very robust manner. In order to predict the locations of the shocks, we have demonstrated a procedure to process the initial velocity configurations data of the particles, and this procedure works equally well also for the one-dimensional inelastic gas [14, 17]. The coefficient of restitution does play a role to decide which of the large jumps in the particles' velocities at early times yield shocks, but at late times, the macroscopic velocity profile for a given model with a given initial configuration of the particles' velocities is inde- pendent of the coefficient of restitution.

Thus, we observe that in terms of detailed structure formation, systems with any $r<1$ "flow" towards the sticky limit $r=0$. Such "universality" has been found in Ref. [17] in terms of global quantities, while our results suggest that a broader "universality" holds for microscopic quantities, such as the locations of shocks (when the same initial velocity configuration is considered).

In addition, for the random lattice model, we have also studied the dynamics of an isolated cluster and clustercluster collisions in detail and obtained an effective "hydrodynamic" equation. We hope that the analyses presented here can be successfully used to study coarsening problems in realistic granular gases (i.e., in two or three dimensions).

S.O. and D.P. are financially supported by the Dutch research organization FOM (Fundamenteel Onderzoek der Materie).
[1] I. Goldhirsch, M.-L. Tan and G. Zanetti, Phys. Rev. Lett. 70, 1619 (1993); J. Sci. Comp. 8, 1 (1993).

[2] S. McNamara and W. R. Young, Phys. Rev. E 50, R28 (1994).

[3] S. McNamara and W. R. Young, Phys. Rev. E 53, 5089 (1996).

[4] J. A. G. Orza, R. Brito, T. P. C. Van Noije and M. H. Ernst, Int. J. Mod. Phys. C 8, 953 (1997).

[5] J. Javier Brey, F. Moreno and M. J. Ruiz-Montero, Phys. Fluids 10, 2965 (1998); 10, 2976 (1998).

[6] R. Soto, M. Mareschal and M. Malek-Mansour, Phys. Rev. E 62, 3836 (2000).

[7] T. P. C. van Noije and M. H. Ernst, in Granular Gases, eds. T. Poschel and S. Luding (Springer, NY, 2001).

[8] J. Javier Brey, M. J. Ruiz-Montero and D. Cubero, Phys. Rev. E 60, 3150 (1999).

[9] T. Pöschel and S. Luding eds., Granular Gases, Lecture Notes in Physics 564 (Springer, Berlin, 2001).

[10] S. Miller and S. Luding, Cluster Growth in Two- and Three-dimensional Granular Gases, cond-mat/0304637

[11] S. Chen, Y. Deng, X. Nie and Y. Tu, Phys. Lett. A 269, 218 (2000).

[12] S. Luding and H. J. Herrmann, Chaos 9, 673 (1999).

[13] S. Hill and G. F. Mazenko, Phys. Rev E 67, 061302
(2003)

[14] L. Frachebourg, Phys. Rev. Lett. 82, 1502 (1999); L. Frachebourg, Ph. A. Martin, J. Piasecki, Physica A 279, 69 (2000); L. Frachebourg, Ph. A. Martin, J. Fluid Mech. 417, 323 (2001)

[15] R. Mikkelsen, M. Versluis, E. Koene, G.-W. Bruggert, D. van der Meer, L. van der Weele, and D. Lohse, Phys. Fluids 14, S14 (2002), http://ojps.aip.org/phf/gallery/pdf/2002/S14_1.pdf.

[16] L. P. Kadanoff, Rev. Mod. Phys. 71, 435 (1999)

[17] E. Ben-Naim,S. Y. Chen, G. D. Doolen and S. Redner, Phys. Rev. Lett. 83, 4069 (1999).

[18] A. Baldassarri, U. Marini Bettolo Marconi, A. Puglisi, Europhys. Lett. 58, 14 (2002).

[19] A. Baldassarri, A. Puglisi, U. Marini Bettolo Marconi, "Kinetic Models of Inelastic Gases", Lecture Notes in Physics 624, 95 (2003) (cond-mat/0302418).

[20] E. Ben-Naim, P. L. Krapivsky, "The Inelastic Maxwell Model", Lecture Notes in Physics 624, 65 (2003) (cond-mat/0301238).

[21] Note that this model does not belong to the Maxwell family since the collision frequency does depend on the incoming velocities. 